\title{
Harbater-Mumford Components and Towers of Moduli Spaces
}

\author{
PierRe DÈbes AND Michel Emsalem
}

\begin{abstract}
A method of choice for realizing finite groups as regular Galois groups over $\mathbb{Q}(T)$ is to find $\mathbb{Q}$-rational points on Hurwitz moduli spaces of covers. In another direction, the use of the so-called patching techniques has led to the realization of all finite groups over $\mathbb{Q}_{p}(T)$. Our main result shows that, under some conditions, these $p$-adic realizations lie on some special irreducible components of Hurwitz spaces (the so-called Harbater-Mumford components), thus connecting the two main branches of the area. As an application, we construct, for every projective system $\left(G_{n}\right)_{n \geq 0}$ of finite groups, a tower of corresponding Hurwitz spaces $\left(\mathcal{H}_{G_{n}}\right)_{n \geq 0}$, geometrically irreducible and defined over $\mathbb{Q}$, which admits projective systems of $\mathbb{Q}_{p}^{\mathrm{ur}}$-rational points for all primes $p$ not dividing the orders $\left|G_{n}\right|(n \geq 0)$.
\end{abstract}

2000 MSC. Primary 12F12 14H30 14H10 ; Secondary 14D15 14G22 32Gxx

\section{Introduction}

Let $\left(G_{n}\right)_{n \geq 0}$ be a projective system of finite groups, given with surjective morphisms $s_{n}: G_{n} \rightarrow G_{n-1}(n>0)$. In [DeDes] was investigated the problem, given a field $k$, of realizing the projective system $\left(G_{n}\right)_{n}$ by a regular tower $K_{0} \subset \cdots \subset K_{n} \subset K_{n+1} \subset \cdots$ of extensions $K_{n} / k(T)$ : that is, $\operatorname{Gal}\left(K_{n} / k(T)\right) \simeq G_{n}$, compatibly with the $s_{n}$ and $K_{n} / k$ is regular $(n \geq 0)$. Constructions of such towers were then notably performed in the case that $k$ is a henselian field containing all roots of 1 of order prime to the residue characteristic $p \geq 0$ of $k$, under the only assumption that each group $G_{n}$ is of order prime to $p$, i.e., is a $p^{\prime}$-group $(n \geq 0)$. As an application, the free profinite group $\widehat{F}_{\omega}$ with countably many generators can be regularly realized as the Galois group of an extension of $\mathbb{Q}^{\text {ab }}((x))(T)$; and similarly, its prime-to- $p$ quotient $\widehat{F}_{\omega}^{\left(p^{\prime}\right)}$ over $\mathbb{Q}_{p}^{\text {ur }}(T)$ (see [DeDes] for more examples).

Using moduli spaces of covers, these problems and results interpret as those of existence of projective systems of $k$-rational points on certain towers $\left(\mathcal{H}_{n}\right)_{n \geq 0}$ of algebraic varieties (given with maps $\mathcal{H}_{n+1} \rightarrow \mathcal{H}_{n}$ ). However the varieties $\mathcal{H}_{n}$ of [DeDes] - some Hurwitz spaces - are reducible in general. Our motivation in the current paper was to obtain a similar result but with the $\mathcal{H}_{n}$ geometrically irreducible and defined over $\mathbb{Q}(n \geq 0)$. 
The key is to use the Harbater-Mumford components of Hurwitz spaces, which have been introduced by Fried [Fr]. Their definition, of topological nature, is recalled in section 1. As explained in [Fr], HM-components can be characterized by the way the covers they carry degenerate. We provide a new approach to this using Wewers' compactification of Hurwitz spaces [We1]. This allows us to show that HM-components are permuted by $\operatorname{Gal}(\overline{\mathbb{Q}} / \mathbb{Q})$, which was proved in $[\mathrm{Fr}]$ under some technical assumptions. We can also prove the following fact, which is a main ingredient of our construction, and which certainly motivated Fried's naming of Harbater-Mumford components: the p-adic covers constructed by Harbater's patching methods [Ha] or by its rigid variants [Li] [Po1] lie on HM-components (under some assumptions). We offer two arguments. One is a deformation argument (from $\mathbb{C}$ to $\mathbb{C}\{\{t\}\}$ ) based on a general "comparison theorem" (proved in [Em2]) expressing the fundamental group of a semi-stable curve in terms of those of the components of the special fiber. Our second argument is $a d$ hoc and is of topological nature (over $\mathbb{C}$ ). Both use Wewers' description of the boundary of Hurwitz spaces.

Our original goal is reached in the final section. To any system $\left(G_{n}\right)_{n \geq 0}$ can be attached a tower $\left(\mathcal{H}_{n}\right)_{n \geq 0}$ of algebraic varieties $\mathcal{H}_{n}$, geometrically irreducible and defined over $\mathbb{Q}$, and which has the following properties (see theorem 4.1 for a full statement):

- each $\mathcal{H}_{n}$ is a component of some moduli space of Galois covers of group $G_{n}$; in particular, if $Z\left(G_{n}\right)=\{1\}, \mathbb{Q}$-rational points $\mathcal{H}_{n}$ yield regular realizations of $G_{n}$ over $\mathbb{Q}(T)(n \geq 0)$. - there exist projective systems of $\mathbb{Q}_{p}^{\text {ur }}$-points, for every $p$ such that all $G_{n}$ are $p^{\prime}$-groups, - there exist projective systems of $\mathbb{Q}^{\mathrm{ab}}((x))$-points, - there exist projective systems of $\mathbb{R}$-points.

The paper is organized as follows. Section 1 presents the main results. Section 2 provides the main tools. Section 3 gives the proofs of the main results. Section 4 is devoted to the motivating application: we show the above result, improving on [DeDes]. Section 5 is an addendum in which we offer an alternate approach to the main result of [DeDes] using formal geometry rather than rigid geometry.

We wish to thank Q. Liu for some helpful comments.

\section{Main results}

1.1. HM-components of Hurwitz spaces. For every integer $r \geq 2$, denote as usual the configuration space for finite subsets of $\mathbb{P}^{1}$ of cardinality $r$ by $\mathcal{U}_{r}$. Given a subset $\mathbf{t} \in \mathcal{U}_{r}(\mathbb{C})$, define a topological bouquet of $\mathbb{P}^{1} \backslash \mathbf{t}$ to be a $r$-tuple $\underline{\gamma}$ of homotopy classes of loops $\gamma_{1}, \ldots, \gamma_{r}$ based at some point $t_{0} \notin \mathbf{t}$ such that: 
$-\gamma_{1}, \ldots, \gamma_{r}$ generate the topological fundamental group $\pi_{1}^{\text {top }}\left(\mathbb{P}^{1}(\mathbb{C}) \backslash \mathbf{t}, t_{0}\right)$ with the single relation $\gamma_{1} \cdots \gamma_{r}=1$,

- $\gamma_{i}$ is a loop revolving once, anticlockwise, about exactly one branch point in $\mathbf{t}$.

We will also use the notion of algebraic bouquet. If $k$ is an algebraically closed field of characteristic 0 (resp. of characteristic $p>0$ ) and $\mathbf{t} \in \mathcal{U}_{r}(k)$, an algebraic bouquet of $\mathbb{P}_{k}^{1} \backslash \mathbf{t}$ is defined to be an $r$-tuple $\underline{\gamma}$ of elements $\gamma_{1}, \ldots, \gamma_{r}$ of the algebraic fundamental group $\pi_{1}\left(\mathbb{P}_{k}^{1} \backslash \mathbf{t}, t_{0}\right)$ (resp. the maximal prime-to-p quotient $\pi_{1}^{\left(p^{\prime)}\right.}\left(\mathbb{P}_{k}^{1} \backslash \mathbf{t}, t_{0}\right)$ of the fundamental group) based at some point $t_{0} \notin \mathbf{t}$ such that:

- $\gamma_{1}, \ldots, \gamma_{r}$ generate $\pi_{1}\left(\mathbb{P}_{k}^{1} \backslash \mathbf{t}, t_{0}\right)$ (resp. $\left.\pi_{1}^{\left(p^{\prime}\right)}\left(\mathbb{P}_{k}^{1} \backslash \mathbf{t}, t_{0}\right)\right)$ with the single relation $\gamma_{1} \cdots \gamma_{r}=1$ - $\gamma_{i}$ is a generator of some inertia group above $t_{i}$ in the maximal algebraic extension of $k(T)$ unramified above $\mathbf{t}, i=1, \ldots, r$.

Given $\mathbf{t} \in \mathcal{U}_{r}(\mathbb{C})$ and a topological bouquet $\underline{\gamma}$ for $\mathbb{P}^{1}(\mathbb{C}) \backslash \mathbf{t}$, the map sending every complex branched cover $f: X \rightarrow \mathbb{P}_{\mathbb{C}}^{1}$ with branch point set $\mathbf{t}$ to the $r$-tuple whose entries are the monodromy permutations of $f^{-1}\left(t_{0}\right)$ associated with the paths $\gamma_{1}, \ldots, \gamma_{s}$, will be denoted by $\mathrm{BCD}_{\gamma}$ (where $\mathrm{BCD}$ stands for "branch cycle description"). We recall the notion of Harbater-Mumford type for covers of $\mathbb{P}^{1}$, which was introduced by M. Fried [Fr2].

Definition $1.1-A$ cover $f$ with branch point set $\mathbf{t}$ is said to be of Harbater-Mumford type (a HM-cover for short) if $r=2 s$ is even and there exists a topological bouquet $\underline{\gamma}$ for $\mathbb{P}^{1}(\mathbb{C}) \backslash \mathbf{t}$ such that $\mathrm{BCD}_{\underline{\gamma}}(f)$ is of the form $\left(g_{1}, g_{1}^{-1}, \ldots, g_{s}, g_{s}^{-1}\right)$.

Fried was interested in the connected components of HM-covers in the associated moduli spaces of covers, the so-called Hurwitz spaces, which we will freely use (see [De], [Em1], [Vo] for presentations and references). Given a finite group $G$, an integer $r \geq 3$, we denote the Hurwitz space of covers of $\mathbb{P}^{1}$ over an algebraically closed field of characteristic 0 , of group $G$ and with $r$ branch points, by $\mathcal{H}_{r, G}$; unless otherwise specified, isomorphisms between two covers $f: X \rightarrow \mathbb{P}^{1}$ and $g: Y \rightarrow \mathbb{P}^{1}$ are isomorphisms $\chi: X \rightarrow Y$ of algebraic curves such that $g \circ \chi=f$.

There are two variants of Hurwitz spaces, depending on whether one is interested - in mere covers, in which case, the covers are not necessarily Galois and $G$ is the monodromy group, given as a subgroup of the symmetric group $S_{d}$ (with $d$ the degree of the covers), or,

- in G-covers, in which case, the covers are Galois covers given with an isomorphism between their automorphism group and the group $G$.

For simplicity, we will not distinguish the notation in these different situations, which, unless otherwise specified, are both covered in this paper. 
Given an (unordered) $r$-tuple $\mathbf{C}=\left(C_{1}, \ldots, C_{r}\right)$ of conjugacy classes of $G$, we let $\mathcal{H}_{r, G}(\mathbf{C})$ be the union of irreducible components of $\mathcal{H}_{r, G}$ whose points correspond to covers with inertia canonical invariant $\mathbf{C}$ : recall that this invariant is the collection $\left(C_{t}\right)_{t}$ of conjugacy classes $C_{t}$ of distinguished generators of inertia groups ${ }^{1}$ above $t$ as $t$ ranges over the branch points of the cover. The spaces $\mathcal{H}_{r, G}$ and $\mathcal{H}_{r, G}(\mathbf{C})$ are (reducible) smooth quasiprojective varieties; they are defined over $\mathbb{Q}$ and $\mathbb{Q}^{\text {ab }}$ respectively (as integral schemes (not necessarily geometrically integral)); furthermore $\mathcal{H}_{r, G}(\mathbf{C})$ is itself defined over $\mathbb{Q}$ if $\mathbf{C}$ is a rational union of conjugacy classes of $G$.

We denote by $\Psi_{r}: \mathcal{H}_{r, G} \rightarrow \mathcal{U}_{r}$ the étale cover mapping each point $[f] \in \mathcal{H}_{r, G}$ to the branch point set $\mathbf{t}$ (of cardinality $r$ ) of the isomorphism class of the cover $f$ that is represented by the point $[f]$. There is a one-one correspondence between each fiber $\Psi_{r}^{-1}(\mathbf{t})$ $\left(\mathbf{t} \in \mathcal{U}_{r}(\mathbb{C})\right)$ and the set

$$
\operatorname{ni}(\mathbf{C})^{\bullet}=\left\{\begin{array}{l|l}
\left(g_{1}, \ldots, g_{r}\right) \in G^{r} & \begin{array}{l}
g_{1} \cdots g_{r}=1 \\
<g_{1}, \ldots, g_{r}>=G \\
g_{i} \in C_{\sigma(i)}, i=1, \ldots, r \text { for some } \sigma \in S_{r}
\end{array}
\end{array}\right\} / \sim
$$

where, by "/ $"$ ", we mean that the tuples $\left(g_{1}, \ldots, g_{r}\right)$ are regarded up to componentwise conjugation by elements of $G$ for G-covers, and, by elements of $\operatorname{Nor}_{S_{d}}(G)$ for mere covers (in which case $\mathrm{ni}(\mathbf{C})^{\bullet}$ is usually denoted by $\mathrm{ni}(\mathbf{C})^{\mathrm{in}}$ or $\mathrm{ni}(\mathbf{C})^{\text {ab }}$ respectively). More specifically, for every topological bouquet $\underline{\gamma}$ of $\mathbb{P}^{1}(\mathbb{C}) \backslash \mathbf{t}$, the map $\mathrm{BCD}_{\underline{\gamma}}$ induces such a one-one correspondence between $\Psi_{r}^{-1}(\mathbf{t})$ and ni $(\mathbf{C})^{\bullet}$. Furthermore, (geometrically irreducible) components of $\mathcal{H}_{r, G}(\mathbf{C})$ correspond to orbits of the action of $\pi_{1}\left(\mathcal{U}_{r}\right)$ on $\Psi_{r}^{-1}(\mathbf{t})$, which classically correspond to orbits of the standard action of the Hurwitz monodromy group $H_{r}$ on the set ni $(\mathbf{C})^{\bullet}$. More precisely, we have this:

(*) A component $X \subset \mathcal{H}_{r, G}(\mathbf{C})(\mathbb{C})$ corresponding to some orbit $\mathcal{O} \subset \operatorname{ni}(\mathbf{C})^{\bullet}$ is the set of those points $[f]$ which have this property: for any $\mathbf{g} \in \mathcal{O}$, there exists a topological bouquet $\underline{\gamma}$ for $\mathbb{P}^{1}(\mathbb{C}) \backslash \Psi_{r}([f])$ such that the branch cycle description $\operatorname{BCD}_{\underline{\gamma}}(f)$ of the cover $f$ is $\mathbf{g}$. Furthermore, given any $\mathbf{t} \in \mathcal{U}_{r}(\mathbb{C})$ and any topological bouquet $\gamma$ for $\mathbb{P}^{1} \backslash \mathbf{t}$, the orbit $\mathcal{O}$ is exactly the set of all branch cycles description $\mathrm{BCD}_{\underline{\gamma}}(f)$ with $[\bar{f}] \in X \cap \Psi_{r}^{-1}(\mathbf{t})$.

Suppose $r=2 s$ and $\mathbf{C}$ consists of $s$ pairs $\left(C_{i}, C_{i}^{-1}\right), i=1, \ldots, s$. Let $\operatorname{HM}(\mathbf{C})$ be the set of all $r$-tuples in $\operatorname{ni}(\mathbf{C})^{\bullet}$ of the form $\mathbf{g}=\left(g_{1}, g_{1}^{-1}, \ldots, g_{s}, g_{s}^{-1}\right)$. These tuples are called $\mathrm{H}($ arbater- $) \mathrm{M}($ umford $)$ representatives of ni $(\mathbf{C})^{\bullet}$ in $[\mathrm{Fr}]$.

1

We assume throughout the paper we have fixed a coherent system $\left(\zeta_{n}\right)_{n>0}$ of roots of unity; the distinguished generator of some inertia group $I$, say of order $e$, is the generator that corresponds to $\zeta_{e}$ in the natural isomorphism between $I$ and the group $\mu_{e}$ of $e$-th roots of 1 . 
Definition $1.2-A H$ (arbater-) $M$ (umford) component of the Hurwitz space $\mathcal{H}_{r, G}(\mathbf{C})$ is the component of some HM-cover. Equivalently, it is a component that corresponds to the orbit of some HM-representative under the action of the Hurwitz braid group $H_{r}$.

All points in a HM-component correspond to HM-covers but in general there may be several HM-components. However, Fried proved the following $[\mathrm{Fr}]$ theorem 3.21. He defines first the notion of g-complete and HM-g-complete tuples $\mathbf{C}$. A tuple $\mathbf{C}$ is g-complete if it satisfies " $g_{i} \in C_{i}, i=1, \ldots, r \Rightarrow<g_{1}, \ldots, g_{r}>=G$ ". A tuple $\mathbf{C}$ with the shape $\left(C_{1}, C_{1}^{-1}, \ldots, C_{s}, C_{s}^{-1}\right)$ is HM-g-complete if it has this property: if any pair $C_{i}, C_{i}^{-1}$ is removed then what remains is g-complete. He then proves that if $\mathbf{C}$ is HM-g-complete, then all HM-representatives are in the same orbit of the Hurwitz braid group $H_{r}$. Consequently, there is then a unique HM-component. Furthermore, if $Z(G)=\{1\}$ and if $\mathbf{C}$ is a rational union of conjugacy classes, then this HM-component is defined over $\mathbb{Q}$. We will re-establish this fact, as a consequence of theorem 1.3, without using the assumption $Z(G)=\{1\}$.

1.2. Boundary of HM-components. Fix as above a finite group $G$, an even integer $r=2 s$ and an $r$-tuple $\mathbf{C}$ consisting of $s$ pairs $\left(C_{i}, C_{i}^{-1}\right), i=1, \ldots, s$. In addition, fix a henselian field $k$, i.e., the fraction field of a discrete valuation ring $\mathcal{O}$, supposed to be henselian. Denote the residue field by $\kappa$ and the characteristic of $\kappa$ by $p$; if $p>0$, we assume that $p$ does not divide $|G|$. We also assume that $k$ is of characteristic 0 and contains all roots of 1 of prime-to- $p$ order (see remark $4.2(\mathrm{~b})$ ). For example, $k$ can be $\mathbb{Q}_{p}^{\text {ur }}$ or $\mathbb{Q}^{\text {ab }}((x))$.

Suppose given a set $\mathbf{t}=\left\{x_{1}, y_{1}, \ldots, x_{s}, y_{s}\right\} \subset \mathbb{P}^{1}(k)$. Assume further that, modulo the maximal ideal $\mathcal{P}$ of $\mathcal{O}$,

$\left(^{*}\right) x_{i}$ and $y_{i}$ are in the same coset, $i=1, \ldots, r$, and,

$x_{1}, \ldots, x_{s}$ lie in pairwise distinct cosets.

(For points $a, b$ in $k$ identified with $\mathbb{P}^{1}(k) \backslash\{\infty\}$, being in the same coset modulo $\mathcal{P}$ more explicitly means that either $|a| \leq 1,|b| \leq 1$ and $|a-b|<1$, or, $|a|>1$ and $|b|>1$ ).

Then $\mathbb{P}_{k}^{1}$ marked by the $r$-points $x_{1}, y_{1}, \ldots, x_{s}, y_{s}$ has a unique stable model $\widetilde{P}_{\mathbf{t}}$ over $\mathcal{O}$ such that the points $x_{1}, y_{1}, \ldots, x_{s}, y_{s}$ extends to sections $\tilde{x}_{1}, \tilde{y}_{1} \ldots, \tilde{x}_{s}, \tilde{y}_{s}$ specializing at distinct points $\bar{x}_{1}, \bar{y}_{1}, \ldots, \bar{x}_{s}, \bar{y}_{s}$ of the special fiber. The special fiber is a comb, i.e., a stable curve of genus 0 marked by $r=2 s$ points with a root $T_{0}$, given with an isomorphism $T_{0} \simeq \mathbb{P}^{1}$, attached to $s$ end components $T_{1}, \ldots, T_{s}$, each of them isomorphic to $\mathbb{P}^{1}$ marked by two points. Denote the intersection point of $T_{i}$ and $T_{0}$ by $\bar{a}_{i}, i=1, \ldots, s$.

We identify $T_{0}$ with the special fiber of $\mathbb{P}_{\mathcal{O}}^{1}$ and call by abuse of language $\bar{a}_{i}$ the specialisation of $x_{i}$ and $y_{i}$ on this special fiber $(i=1, \ldots, r)$. The following diagram summarizes the situation 


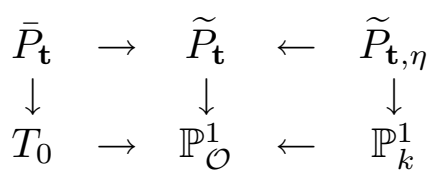

where $\bar{P}_{\mathbf{t}}$ denotes the special fiber of $\widetilde{P}_{\mathbf{t}}$ and where the last vertical arrow is an isomorphism between the generic fibers.

For each index $i=1, \ldots, r$, we introduce the disk $D_{i}$ which is the set of points of $\mathbb{P}_{k}^{1}$ which reduce to $\bar{a}_{i}$ in the special fiber $T_{0}$, we denote by $\omega_{i}$ a point of $D_{i}$ such that $\left|x_{i}-\omega_{i}\right|=\left|y_{i}-\omega_{i}\right|=\left|x_{i}-y_{i}\right|$ and by $\partial D_{i}$ the set of points $x$ of $D_{i}$ satisfying the relation $\left|x-\omega_{i}\right|>\left|x_{i}-\omega_{i}\right|=\left|y_{i}-\omega_{i}\right|$. The model $\widetilde{P}_{\mathbf{t}}$ is obtained from $\mathbb{P}_{\mathcal{O}}^{1}$ by a sequence of blowing up at the points $\bar{a}_{1}, \ldots, \bar{a}_{r}$ (viewed as points of the special fiber of $\mathbb{P}_{\mathcal{O}}^{1}$ ). The fiber of $\bar{a}_{i}$ in the natural morphism $\widetilde{P}_{\mathbf{t}} \rightarrow \mathbb{P}_{\mathcal{O}}^{1}$ is the exceptional component $T_{i}$, the points of $\partial D_{i}$ specialize to $\bar{a}_{i}$ and those of $D_{i} \backslash \partial D_{i}$ specialize to points of $T_{i}-\left\{\bar{a}_{i}\right\}(i=1, \ldots, r)$.

Consider the compactification $\overline{\mathcal{H}_{r, G}(\mathbf{C})}$ of $\mathcal{H}_{r, G}(\mathbf{C})$ constructed by S. Wewers [We1]. The natural étale morphism $\Psi_{r}: \mathcal{H}_{r, G} \rightarrow \mathcal{U}_{r}$ extends to a ramified cover $\overline{\mathcal{H}_{r, G}(\mathbf{C})} \rightarrow \overline{\mathcal{U}}_{r}$. Points on the boundary $\overline{\mathcal{U}}_{r} \backslash \mathcal{U}_{r}$ represent stable marked curves of genus 0 with a root, i.e. trees of curves of genus 0 with a distinguished component $T_{0}$ - the root - equipped with an isomorphism $\mathbb{P}^{1} \simeq T_{0}$ and at least three marked points (including the double point) on any component but the root [We1] (e.g. combs as above). Points on the boundary $\overline{\mathcal{H}_{r, G}(\mathbf{C})} \backslash \mathcal{H}_{r, G}(\mathbf{C})$ represent admissible covers of stable marked curves $B$ of genus 0 with root [We1]. We briefly explain what is an admissible cover over some field $\kappa$ with the tools introduced above.

It is a finite morphism $Y \rightarrow B$ over $\kappa$, with $Y$ connected, whose restriction above each irreducible component of $B$ is a branched cover and which satisfies the following local condition above each double point of $B$. Let $\mathcal{O}$ be a discrete valuation ring with residue field $\kappa$ and $\widetilde{B}$ be the unique extension of $B$ over $\mathcal{O}$. A formal neighborhood of the double points of $B$ in $\widetilde{B}$ is of the form $\widetilde{Z}=\operatorname{Spec}(\mathcal{O}[[s, t]] /(s t-\pi)$ where $\pi$ is in the maximal ideal of $\mathcal{O}$. The special fiber of $\widetilde{Z}$ has two components $Z_{1}, Z_{2}$, and restriction induces equivalences of categories between the category of $p^{\prime}$-covers of the geometric generic fiber $\widetilde{Z}_{\bar{\eta}}$ and the category of $p^{\prime}$-covers of $\bar{Z}_{1}=Z_{1} \times_{\operatorname{Spec}(\kappa)} \operatorname{Spec}(\bar{\kappa})\left(\operatorname{resp} . \bar{Z}_{2}=Z_{2} \times_{\operatorname{Spec}(\kappa)} \operatorname{Spec}(\bar{\kappa})\right)$ unramified outside the double point ${ }^{2}$. An admissible cover of the special fiber is a collection of $p^{\prime}$-covers of the components of the special fiber such that at any double point, the two $p^{\prime}$-covers of $\widetilde{Z}_{\bar{\eta}}$ obtained via the equivalences of categories introduced above, are isomorphic.

2 These categories are Galois categories with fundamental group $\widehat{\mathbb{Z}}^{p^{\prime}}$, the maximal prime-to-p quotient of $\widehat{\mathbb{Z}}$. 
Below we call HM-admissible cover every admissible cover of a comb that is unramified at the singular points. Over $\mathbb{C}$, this corresponds to a finite topological (unramified) cover of a complex comb with each pair of marked points removed from the end components.

Theorem 1.3 - The HM-components of $\mathcal{H}_{r, G}(\mathbf{C})$ are those components whose boundary in $\overline{\mathcal{H}_{r, G}(\mathbf{C})}$ contains points representing HM-admissible covers.

It follows from this characterization that if $\mathcal{H}$ is some HM-component of $\mathcal{H}_{r, G}(\mathbf{C})$, then for every $\tau \in \operatorname{Gal}(\overline{\mathbb{Q}} / \mathbb{Q}), \mathcal{H}^{\tau}$ is a HM-component of $\mathcal{H}_{r, G}(\mathbf{C})^{\tau}$. Consequently, if there is a unique HM-component $\mathcal{H} \subset \mathcal{H}_{r, G}(\mathbf{C})$ and if $\mathbf{C}$ is a rational union of conjugacy classes of $G$ (which yields $\mathcal{H}_{r, G}(\mathbf{C})^{\tau}=\mathcal{H}_{r, G}(\mathbf{C})$ ), then $\mathcal{H}$ is defined over $\mathbb{Q}$.

1.3. Construction of HM-covers from patching methods. We retain the notation and assumptions from $\S 1.2$, notably those regarding the set $\mathbf{t}=\left\{x_{1}, y_{1} \ldots, x_{s}, y_{s}\right\}$. In addition we assume here $k$ is complete. Suppose given a $k$-cover $f: X \rightarrow \mathbb{P}_{k}^{1}$ of group $G$, with branch point set $\mathbf{t}$ and with corresponding inertia canonical invariant $\mathbf{C}$ (consisting as above of $s$ pairs $\left.\left(C_{i}, C_{i}^{-1}\right), i=1, \ldots, s\right)$. Recall the assumption $p \nmid|G|$ (if $p>0$ ), that is, $f$ is a $p^{\prime}$-cover. The cover $f: X \rightarrow \mathbb{P}_{k}^{1}$, after a possible finite extension of $k$, uniquely extends to a cover $\tilde{f}: \widetilde{X} \rightarrow \widetilde{P}_{\mathbf{t}}$. Its special fiber $\bar{f}: \bar{X} \rightarrow \bar{P}_{\mathbf{t}}$ is branched at the marked points on the end components and also possibly at the singular points $\bar{a}_{1}, \ldots, \bar{a}_{s}$. Denote also by $f_{i}, i=1, \ldots, s$, the restricted rigid cover $f$ above the disk $D_{i}$ previously defined.

Proposition $\mathbf{1 . 4}$ - The following conditions are equivalent.

(i) each restricted cover $f_{i}$ is trivial above $\partial D_{i}, i=1, \ldots, s$,

(ii) each restricted cover $f_{i}$ extends to a cover $g_{i}: Y_{i} \rightarrow \mathbb{P}^{1}$ with only two branch points ${ }^{3}$ $\left(x_{i}\right.$ and $\left.y_{i}\right), i=1, \ldots, s$,

(iii) the special fiber $\bar{f}$ of $\tilde{f}$ is unramified at the singular points $\bar{a}_{1}, \ldots, \bar{a}_{s}$ of the comb $\bar{P}_{\mathbf{t}}$, that is, $\bar{f}$ is a HM-admissible cover.

Let $\mathbb{Q}(\mathbf{t}) \subset k$ be the subfield generated by the branch point set $\mathbf{t}=\left\{x_{1}, y_{1}, \ldots, x_{s}, y_{s}\right\}$ of the cover $f$ and $\overline{\mathbb{Q}(\mathbf{t})} \subset \bar{k}$ be its algebraic closure inside $\bar{k}$. It classically follows (from Riemann's existence theorem or Grothendieck's specialization theorem) that $f$ can be defined over $\overline{\mathbb{Q}(\mathbf{t})}$. Next fix a $\overline{\mathbb{Q}}$-embedding $i: \overline{\mathbb{Q}(\mathbf{t})} \hookrightarrow \mathbb{C}$. Via this embedding, the cover $f$ induces a $\mathbb{C}$-cover $f^{i}: X^{i} \rightarrow \mathbb{P}_{\mathbb{C}}^{1}$ of group $G$, with branch point set $\mathbf{t}^{i}$ and with inertia

$3 g_{i}$ is then necessarily a cyclic cover of $\mathbb{P}^{1}$ by a curve $Y_{i}$ of genus 0. 
canonical invariant $\mathbf{C}$. Denote the corresponding complex point in $\mathcal{H}_{r, G}(\mathbf{C})$ by $[f]^{i}$; it is the image via $i$ of the $k$-point $[f] \in \mathcal{H}_{r, G}(k)$. We obtain the following result as a consequence of theorem 1.3 and proposition 1.4 .

Corollary $\mathbf{1 . 5}$ - If the cover $f$ satisfies the equivalent conditions of proposition 1.4, then the point $[f]^{i}$ lies in a HM-component of $\mathcal{H}_{r, G}(\mathbf{C})$.

Denote the irreducible component of $[f]$ by $\mathcal{H}$. It is defined over $\overline{\mathbb{Q}}$ (and over $k$ ) and so the statement above does not depend on the $\overline{\mathbb{Q}}$-embedding $i: \overline{\mathbb{Q}(\mathbf{t})} \hookrightarrow \mathbb{C}$.

Remark 1.6. Using patching techniques from formal analytic geometry, Harbater proved that, for fields $k$ as above, each finite group can be realized as the automorphism group of a $k$-G-cover of $\mathbb{P}_{k}^{1}$ [Ha]. In this remark, we explain that for $p^{\prime}$-groups, the realizing covers can be required to further satisfy the conclusions of proposition 1.4 and so of corollary 1.5. This remark will be used in section 4 to construct projective systems of $k$-rational points on infinite towers of Hurwitz spaces.

After Harbater's original paper, other proofs using rigid analytic geometry (rather than formal) were given by Liu [Li] and Pop [Po1] [Po2]. Given a $p^{\prime}$-group $G$ and a $r$-tuple $\mathbf{C}$ as above, they can construct a $k$-G-cover of group $G$ with inertia canonical invariant $\mathbf{C}$. Furthermore, this cover satisfies condition (i) from proposition 1.4. The patching methods they use however impose some restriction on the branch point set $\mathbf{t}=\left\{x_{1}, y_{1} \ldots, x_{s}, y_{s}\right\} \subset$ $\mathbb{P}^{1}(k)$; they have this starting hypothesis:

$(* *)\left|x_{i}-y_{i}\right|<\left|x_{i}-x_{j}\right||p|^{\frac{1}{p-1}}, j \neq i, i=1, \ldots, s$

(with the convention that $|p|^{\frac{1}{p-1}}=1$ in the case $p=0$ ).

If one picks the points $x_{1}, y_{1} \ldots, x_{s}, y_{s} \in \mathbb{P}^{1}(k)$ satisfying both conditions $(*)$ from $\S 1.2$ and $\left(^{* *}\right)$ above $\left(e . g .\left|x_{i}\right|=1,\left|x_{i}-x_{j}\right|=1\right.$ and $\left.\left|x_{i}-y_{i}\right|<|p|^{\frac{1}{p-1}}(i, j \in\{1, \ldots, s\}, i \neq j)\right)$, then the construction leads to covers satisfying the conclusions of proposition 1.4.

Alternatively, one can, as in [Ha] or [Mo] use formal geometry. With $\mathbf{t}=\left\{x_{1}, y_{1} \ldots, x_{s}, y_{s}\right\}$ as in $\S 1.2$, one starts from admissible covers $\bar{f}$ of the special fiber $\bar{P}_{\mathbf{t}}$ such that (i) the cover $\bar{f}$ restricted to each end component $T_{i}$ is a cyclic cover of group $<g_{i}>\subset G$ branched at two points distinct from the intersection point of $T_{i}$ with the root $T_{0}$, and with inertia canonical invariant $\left(g_{i}, g_{i}^{-1}\right), i=1, \ldots, s$,

(ii) $\bar{f}$ is a trivial cover above $\mathbb{P}_{\kappa}^{1} \backslash\left\{\bar{a}_{1}, \ldots, \bar{a}_{s}\right\}$.

Condition (i) implies that $\bar{f}$ is a HM-admissible cover. Such an admissible cover extends to a $k$-cover $f: X \rightarrow \mathbb{P}_{k}^{1}$ of group $G$, with branch point set $\mathbf{t}$ (see [Ha], [Mo], [We1], [We2]). 
The fact that one can also prescribe the inertia canonical invariant $\mathbf{C}$ follows from the comparison theorem from $\S 2.1$. We will adopt this point of view in $\S 5$.

\section{Tools}

2.1. Comparison theorem of fundamental groups. A main tool in the proof of theorem 1.3 is a comparison theorem between the fundamental groups of the generic fiber and of the components of the special fiber of a stable marked curve.

The situation is the following. We are given a stable marked curve $Z$ over the valuation ring $\mathcal{O}$ of a henselian field $k$. We assume the residue field $\kappa$ to be algebraically closed. We shall only consider here the special case where $Z$ is of genus 0 and its special fiber is a comb. We denote its root by $T_{0}$, its end components by $T_{1}, \ldots, T_{s}$, the intersection point of $T_{0}$ with $T_{i}$ by $\bar{a}_{i}$ and the marked points on $T_{i}$ by $\bar{x}_{i}, \bar{y}_{i}, i=1, \ldots, s$. We also denote by $\left\{x_{1}, y_{1}, \ldots x_{s}, y_{s}\right\}$ the marked points on the generic fiber $Z_{\eta}$, which extend to sections $\left\{\tilde{x}_{1}, \tilde{y}_{1}, \ldots, \tilde{x}_{s}, \tilde{y}_{s}\right\}$ on $Z$. There are natural restriction functors from the category of $p^{\prime}$-covers of the geometric generic fiber $Z_{\bar{\eta}}$ to the category of $p^{\prime}$-covers of $T_{i}, i=1, \ldots, s$. These functors induce morphisms

$$
\begin{aligned}
& \theta_{i}: \pi_{1}\left(T_{i} \backslash\left\{\bar{x}_{i}, \bar{y}_{i}, \bar{a}_{i}\right\}, \xi_{i}\right) \rightarrow \pi_{1}\left(Z_{\bar{\eta}} \backslash\left\{x_{1}, y_{1}, \ldots, x_{s}, y_{s}\right\}, \xi\right) \quad(i=1, \ldots, s) \\
& \theta_{0}: \pi_{1}\left(T_{0} \backslash\left\{\bar{a}_{1}, \ldots, \bar{a}_{s}\right\}, \xi_{0}\right) \rightarrow \pi_{1}\left(Z_{\bar{\eta}} \backslash\left\{x_{1}, y_{1}, \ldots, x_{s}, y_{s}\right\}, \xi\right)
\end{aligned}
$$

defined up to conjugation and where $\xi_{0}, \xi_{1}, \ldots \xi_{s}$ and $\xi$ are appropriate base points.

\section{Theorem 2.1 — Then there exist}

- an algebraic bouquet $\gamma^{(0)}=\left\{\gamma_{1}^{(0)}, \ldots, \gamma_{s}^{(0)}\right\}$ for $T_{0} \backslash\left\{\bar{a}_{1}, \ldots, \bar{a}_{s}\right\}$ based at $\xi_{0}$,

- an algebraic bouquet $\underline{\gamma}^{(i)}=\left\{\gamma_{0}^{(i)}, \gamma_{1}^{(i)}, \gamma_{2}^{(i)}\right\}$ for $T_{i} \backslash\left\{\bar{a}_{i}, \bar{x}_{i}, \bar{y}_{i}\right\}$ based at $\xi_{i}, i=1, \ldots, s$, and

- elements $\sigma_{i} \in \pi_{1}\left(Z_{\bar{\eta}} \backslash\left\{x_{1}, y_{1}, \ldots, x_{s}, y_{s}\right\}, \xi\right), i=1, \ldots, s$, such that $\pi_{1}\left(Z_{\bar{\eta}} \backslash\left\{x_{1}, y_{1}, \ldots, x_{s}, y_{s}\right\}, \xi\right)$ is generated by the elements

- $\theta_{0}\left(\gamma_{1}^{(0)}\right), \ldots, \theta_{0}\left(\gamma_{s}^{(0)}\right)$, and

- $\theta_{i}\left(\gamma_{0}^{(i)}\right), \theta_{i}\left(\gamma_{1}^{(i)}\right), \theta_{i}\left(\gamma_{2}^{(i)}\right), i=1, \ldots, s$

with the only relations $\theta_{0}\left(\gamma_{i}^{(0)}\right) \cdot \theta_{i}\left(\gamma_{0}^{(i)}\right)^{\sigma_{i}}=1, i=1, \ldots, s$.

We shall use in the proof of lemma 3.2 the topological version of this theorem: if the residue field $\kappa$ is $\mathbb{C}$, and if one replaces algebraic fundamental groups by topological fundamental groups and bouquets by topological bouquets, the statement of Theorem 2.1 holds. The algebraic fundamental groups are the profinite completions of the topological 
fundamental groups, and the morphisms $\theta_{i}$ are induced by the $\theta_{i}^{\text {top }}$ (see first proof of lemma 3.2 for a more precise statement).

2.2. HM-covers degenerating to HM-admissible covers. The general construction below, which shows some HM-covers degenerate to HM-admissible covers (over $\mathbb{C}$ ), will be used in the proof of theorem 1.3.

Let $S^{2} \subset \mathbb{R}^{3}$ be the unit sphere (identified with $\mathbb{P}^{1}(\mathbb{C})$ ) and let $\mathbf{t}=\left\{x_{1}, y_{1}, \ldots, x_{s}, y_{s}\right\} \subset$ $S^{2}$ be a subset of $r=2 s$ distinct points. Suppose also given $s$ open disks $U_{1}, \ldots, U_{s}$ such that $\overline{U_{i}} \cap \overline{U_{j}}=\emptyset$ and $x_{i}, y_{i} \in U_{i}$, and pick a point $a_{i}$ on the line segment $\left[x_{i}, y_{i}\right]$, $(i, j=1, \ldots, s$ and $i \neq j)$.

Consider the continuous deformation $\mathbf{t}^{\theta}=\left\{x_{1}^{\theta}, y_{1}^{\theta}, \ldots, x_{s}^{\theta}, y_{s}^{\theta}\right\}$ parametrized by $\theta \in[0,1]$ of the marking $\mathbf{t}=\mathbf{t}_{0}$ given by

$$
\left\{\begin{array}{l}
x_{i}^{\theta}=(1-\theta) x_{i}+\theta a_{i} \\
y_{i}^{\theta}=(1-\theta) y_{i}+\theta a_{i}
\end{array} \quad(i=1, \ldots, s)\right.
$$

This deformation induces a continuous path between the representing points on the moduli space $\overline{\mathcal{U}_{r}}$. In Wewers' modular compactification of $\mathcal{U}_{r}$, the limit point (for $\theta=1$ ) represents a comb. This comb is obtained by blowing up the deformation space $\mathbf{t}^{\theta}(\theta \in[0,1])$ at each double point $x_{i}^{1}=y_{i}^{1}, i=1, \ldots, s$ (see figures for a topological representation of this process). Denote the resulting comb by $\mathcal{C}$, which is the union of the sphere $S^{2}$ with $s$ "small" spheres $\Sigma_{1}, \ldots, \Sigma_{s}$, pairwise disjoint, attached to $S^{2}$ at the points $a_{1}, \ldots, a_{s}$ respectively and marked by two distinct points (distinct from $a_{1}, \ldots, a_{s}$ ).

For each $i=1, \ldots, s$, let $\gamma_{i, 1}, \gamma_{i, 2}$ be closed paths based at $a_{i}$, revolving around the segment line $\left[x_{i}, a_{i}\right]$ and $\left[a_{i}, y_{i}\right]$; for each $\theta \in[0,1[$, their homotopy classes freely generate $\pi_{1}^{\text {top }}\left(U_{i} \backslash\left\{x_{i}^{\theta}, y_{i}^{\theta}\right\}, a_{i}\right)$. Fix a point $a_{0} \in S^{2} \backslash \bigcup_{1 \leq i \leq s} \overline{U_{i}}$ and a set of paths $\delta_{1}, \ldots, \delta_{s}$, pairwise disjoint and connecting $a_{0}$ to $a_{1}, \ldots, a_{s}$ respectively, in such a way that, setting $\widetilde{\gamma}_{i, j}=\delta_{i} \gamma_{i, j} \delta_{i}^{-1}(i=1, \ldots, s, j=1,2)$, the paths $\widetilde{\gamma}_{1,1}, \widetilde{\gamma}_{1,2}, \ldots, \widetilde{\gamma}_{s, 1}, \widetilde{\gamma}_{s, 2}$ constitute a topological bouquet $\tilde{\gamma}$ for each base space $S^{2} \backslash \mathbf{t}^{\theta}$ based at $a_{0}(\theta \in[0,1[)$.

Next let $d \geq 1$ be an integer and $G \subset S_{d}$ be a subgroup of $S_{d}$ given with a generating system $\left\{g_{1}, \ldots, g_{s}\right\}$. For every $\theta \in\left[0,1\left[\right.\right.$, let $\phi_{\theta}: \pi_{1}^{\text {top }}\left(S^{2} \backslash \mathbf{t}^{\theta}, a_{0}\right) \rightarrow G \subset S_{d}$ be the epimorphism mapping $\widetilde{\gamma}_{i, 1}$ to $g_{i}$ and $\widetilde{\gamma}_{i, 2}$ to $g_{i}^{-1}, i=1, \ldots, s$. Denote the associated $\mathbb{C}$ cover by $f_{\theta}$ and the corresponding representing point on $\mathcal{H}_{r, G}$ by $h_{\theta}$. By construction, the covers $f_{\theta}$ are those obtained from $f_{0}$ by the deformation $\mathbf{t}^{\theta}(\theta \in[0,1[)$. The first part of the lemma below also follows by construction. 
Lemma 2.2 - The covers $f_{\theta}$ are HM-covers $(\theta \in[0,1[)$. Furthermore, the collection of points $h_{\theta}=\left[f_{\theta}\right]$ converges in $\overline{\mathcal{H}_{r, G}}(\mathbb{C})$ as $\theta \rightarrow 1$ and the limit point $h_{1}$ corresponds to the isomorphism class of a HM-admissible cover $f_{1}$ of the comb $\mathcal{C}$ with cyclic restriction of inertia canonical invariant $\left\{g_{i}, g_{i}^{-1}\right\}$ above each sphere $\Sigma_{i}, i=1, \ldots, s$.

For the second part, consider a sequence $\left(\theta_{n}\right)_{n>0}$ of elements in $[0,1[$ such that the sequence $\left(h_{\theta_{n}}\right)_{n>0}$ converges in $\overline{\mathcal{H}_{r, G}}(\mathbb{C})$. Due to the continuity of $\overline{\mathcal{H}_{r, G}}(\mathbb{C}) \rightarrow \overline{\mathcal{U}_{r}}(\mathbb{C})$, the limit point $h_{1}$ corresponds to the isomorphism class of a cover $f_{1}$ of the comb $\mathcal{C}$.

Set $\mathcal{B}^{\prime}=S^{2} \backslash \bigcup_{i=1}^{s} \overline{U_{i}}$ and fix a path $\beta_{i}$ in $\mathcal{B}^{\prime}$ based at $a_{0}$ revolving around $\overline{U_{i}}, i=$ $1, \ldots, s$, in such a way that the homotopy classes $\left[\beta_{1}\right], \ldots,\left[\beta_{s}\right]$ generate $\pi_{1}^{\text {top }}\left(\mathcal{B}^{\prime}, a_{0}\right)$ with the single relation $\left[\beta_{1}\right] \cdots\left[\beta_{s}\right]=1$. For every $\theta \in[0,1]$, denote by $\phi_{\theta}^{\prime}$ the representation $\pi_{1}^{\text {top }}\left(\mathcal{B}^{\prime}, a_{0}\right) \rightarrow S_{d}$ associated with the restriction $f_{\theta}^{\prime}$ to $\mathcal{B}^{\prime}$ of the cover $f_{\theta}(\theta \in[0,1])$.

For $\theta \in\left[0,1\left[, \phi_{\theta}^{\prime}\right.\right.$ is the restriction of $\phi_{\theta}$ to $\pi_{1}^{\text {top }}\left(\mathcal{B}^{\prime}, a_{0}\right)$. As in $\pi_{1}^{\text {top }}\left(S^{2} \backslash \mathbf{t}^{\theta}, a_{0}\right)$ we have $\left[\beta_{i}\right]=\left[\widetilde{\gamma}_{i, 1}\right]\left[\widetilde{\gamma}_{i, 2}\right]$, the definition of $\phi_{\theta}$ yields $\phi_{\theta}\left(\left[\beta_{i}\right]\right)=1, i=1, \ldots, s$, for all $\theta \in[0,1[$. It follows that $\phi_{\theta}^{\prime}\left(\left[\beta_{i}\right]\right)=1$ in $\pi_{1}^{\text {top }}\left(\mathcal{B}^{\prime}, a_{0}\right), i=1, \ldots, s$, and so, $\phi_{\theta}^{\prime}=1$, for all $\theta \in[0,1[$.

Now the assumption $\lim _{n \rightarrow \infty} h_{\theta_{n}}=h_{1}$ implies that $\phi_{1}^{\prime}=\phi_{\theta_{n}}^{\prime}=1$ (for all $n>0$ ). Therefore the restriction of $f_{1}$ to $S^{2} \backslash \mathbf{t}^{1}$ (the root of the comb $\mathcal{C}$ ) is unramified at the points $a_{1}, \ldots, a_{s}$. This also shows that the restriction of $f_{1}$ to each sphere $\Sigma_{i}$ (each end component) is unramified at $a_{i}$, hence is a cyclic cover branched at two points, $i=1, \ldots, s$. Finally computing the monodromy with respect to the paths $\gamma_{i, 1}$ and $\gamma_{i, 2}$, viewed on the modular presentation of $S^{2} \backslash \mathbf{t}^{\theta}(\theta \in[0,1])$, yields the inertia canonical invariant $\left\{g_{i}, g_{i}^{-1}\right\}$ of $f_{1}$ above each sphere $\Sigma_{i}, i=1, \ldots, s$, and concludes the proof of Lemma 2.2.

Addendum to construction. In the proof of lemma 3.2, we will have to use that

$\left(^{*}\right)$ the construction above can be achieved with the extra constraint that the comb $\mathcal{C}$ and the cover $f_{1}$ are prescribed in advance.

That is, the following will be given: the group $G \subset S_{d}$, the points $a_{1}, \ldots, a_{s}$, and, for $i=1, \ldots, s$, the (not necessarily transitive) representation $\pi_{1}^{\text {top }}\left(\Sigma_{i} \backslash\{2 \mathrm{pts}\}\right) \rightarrow S_{d}$ (for some fixed $d>0$ ) corresponding to the restriction of $f_{1}$ to $\Sigma_{i}$. This last part of the data readily provides an $r$-tuple $\left(g_{1}, g_{1}^{-1}, \ldots, g_{s}, g_{s}^{-1}\right)$ : take for $g_{i}, g_{i}^{-1}$ the images of two standard generators of $\pi_{1}^{\text {top }}\left(\Sigma_{i} \backslash\{2 \mathrm{pts}\}\right), i=1, \ldots, s$. From this, one easily forms an $r$ tuple $\mathbf{t}$, a deformation $\mathbf{t}^{\theta}$ and a cover $f_{0}$ as above such that the corresponding deformation of $f_{0}$ for $\theta=1$ is the prescribed cover $f_{1}$. Furthermore, the (topological) monodromy groups of the covers $f_{0}$ and $f_{1}$ are the same, namely the group $G$ generated by $g_{1}, \ldots, g_{s}$, and the inertia canonical invariant of $f_{0}$ is the $r$-tuple $\mathbf{C}$ consisting of the conjugacy classes in $G$ of $g_{1}, g_{1}^{-1} \ldots, g_{s}, g_{s}^{-1}$. 


\section{Proofs}

3.1. Proof of proposition 1.4. Fix $i \in\{1, \ldots, s\}$. There is a reduction functor from the category of $p^{\prime}$-rigid analytic covers of $D_{i}$, unramified outside $\left\{x_{i}, y_{i}\right\}$ to the category of $p^{\prime}$-algebraic covers of $T_{i}$ unramified outside $\left\{\bar{a}_{i}, \bar{x}_{i}, \bar{y}_{i}\right\}$, and this functor induces a morphism of fundamental groups

$$
F_{i}: \pi_{1}\left(T_{i} \backslash\left\{\bar{a}_{i}, \bar{x}_{i}, \bar{y}_{i}\right\}\right) \rightarrow \pi_{1}^{\mathrm{rig}}\left(D_{i} \backslash\left\{x_{i}, y_{i}\right\}\right)
$$

On the other hand the inclusion induces a morphism

$$
G_{i}: \pi_{1}^{\mathrm{rig}}\left(D_{i} \backslash\left\{x_{i}, y_{i}\right\}\right) \rightarrow \pi_{1}^{\mathrm{rig}}\left(\mathbb{P}^{1} \backslash\left\{x_{1}, y_{1}, \ldots, x_{s}, y_{s}\right\}\right)
$$

On the algebraic side (see $\S 2.1$ ), there are natural morphisms

$$
\theta_{i}: \pi_{1}\left(T_{i} \backslash\left\{\bar{a}_{i}, \bar{x}_{i}, \bar{y}_{i}\right\}\right) \rightarrow \pi_{1}\left(\mathbb{P}^{1} \backslash\left\{x_{1}, y_{1}, \ldots, x_{s}, y_{s}\right\}\right)
$$

Finally the functor which associates to every $p^{\prime}$-algebraic cover of $\mathbb{P}^{1} \backslash\left\{x_{1}, y_{1}, \ldots, x_{s}, y_{s}\right\}$ the corresponding rigid analytic cover, which is an equivalence of categories, induces an isomorphism

$$
H: \pi_{1}^{\mathrm{rig}}\left(\mathbb{P}^{1} \backslash\left\{x_{1}, y_{1}, \ldots, x_{s}, y_{s}\right\}\right) \rightarrow \pi_{1}\left(\mathbb{P}^{1} \backslash\left\{x_{1}, y_{1}, \ldots, x_{s}, y_{s}\right\}\right)
$$

We shall use the following equality (true up to conjugation)

$$
\theta_{i}=H \circ G_{i} \circ F_{i}
$$

(i) $\Rightarrow$ (iii). As explained above, in order to obtain the restriction of $\widetilde{f}$ to the exceptional component $T_{i}$, one can reduce modulo an uniformizing parameter the rigid analytic cover $f_{i}$. The restriction of $f_{i}$ to $\partial D_{i}$ is supposed to be trivial, and the fiber of $\bar{a}_{i}$ in the restriction of $\tilde{f}$ to $T_{i}$ corresponds to the fiber of $\partial D_{i}$ in $f_{i}$. This shows that this restriction is unramified at $\bar{a}_{i}$.

(iii) $\Rightarrow$ (ii). We suppose the restriction $\bar{f}_{i}$ of $\bar{f}$ to $T_{i}$ to be unramified at $\bar{a}_{i}$. So $f_{i}$ extends to a cover of $\mathbb{P}_{\mathcal{O}}^{1}$ unramified outside two points $x_{i}, y_{i}$. The generic fiber of this cover induces a rigid analytic cover $g_{i}: Y_{i} \rightarrow \mathbb{P}^{1}$ unramified outside $\left\{x_{i}, y_{i}\right\}$, whose restriction to $D_{i}$ can be identified to $f_{i}$.

(ii) $\Rightarrow$ (i). Suppose that $f_{i}$ extends to a cover $g_{i}: Y_{i} \rightarrow \mathbb{P}^{1}$ unramified outside $\left\{x_{i}, y_{i}\right\}$. The restriction of $g_{i}$ to any disk containing neither $x_{i}$ nor $y_{i}$ is trivial. So the restriction of $f_{i}$ to $\partial D_{i}$ is trivial. 


\subsection{Proof of theorem 1.3.}

3.2.1. Direct part. Fix $s$ open disks $U_{1}, \ldots, U_{s}$ in $\mathbb{P}^{1}(\mathbb{C})$, pairwise disjoint, and pick distinct points $x_{i}, y_{i}$ in $U_{i}, i=1, \ldots, s$. Set $\mathbf{t}=\left\{x_{1}, y_{1} \ldots, x_{s}, y_{s}\right\}$ and fix a topological bouquet $\tilde{\gamma}$ for $\mathbb{P}^{1} \backslash \mathbf{t}$ as in $\S 2.2$. From $\S 1$.1, if $\mathcal{H}$ is any HM-component of $\mathcal{H}_{r, G}(\mathbf{C})$, there exists an isomorphism class of cover $\left[f_{o}\right] \in \mathcal{H}$ with branch point set $\mathbf{t}$ and with branch cycle description relative to $\underline{\tilde{\gamma}}$ of the form $\left(g_{1}, g_{1}^{-1}, \ldots, g_{s}, g_{s}^{-1}\right)$. The construction given in $\S 2.2$ then applies to show that $\mathcal{H}$ has HM-admissible covers in its boundary.

Alternatively theorem 2.1 can be used to prove this direct part. The rest of the proof is devoted to the converse.

Suppose given a component $\mathcal{H}$ of $\mathcal{H}_{r, G}(\mathbf{C})$ whose boundary contains a point representing a HM-admissible cover $\varphi$ defined over some field $\kappa$. We shall describe "a path in the closure $\overline{\mathcal{H}}$ " from the point representing $\varphi$ to a complex point representing a Harbater-Mumford cover. If $\kappa$ is of characteristic 0 , the first stage can be skipped.

3.2.2. First stage. Suppose that the HM-admissible cover $\varphi$ is defined over a field $\kappa$ of characteristic $p>0$. Let $k$ be a henselian field of characteristic 0 and of residue field $\kappa$. Call $\mathcal{O}$ the ring of integers of $k$.

Lemma 3.1 - The $\kappa$-cover $\varphi$ extends to a HM-admissible cover $\tilde{\varphi}$ of a comb with s end components $\tilde{T}_{1}, \ldots, \tilde{T}_{s}$, each of them being a copy of $\mathbb{P}_{\mathcal{O}}^{1}$, and satisfying the following:

- the restricted cover $\tilde{\varphi}$ above $\tilde{T}_{i}$ is a (not necessarily connected) cyclic cover of group $<g_{i}>\subset G$, branched at two points and unramified at the intersection of $\tilde{T}_{i}$ and the root $\tilde{T}_{0}$, and with inertia canonical invariant $\left(g_{i}, g_{i}^{-1}\right), i=1, \ldots, s$,

- the restricted cover $\tilde{\varphi}$ above the root $\tilde{T}_{0}$ is trivial.

Proof. The base space of the cover $\varphi$ is a comb $\tau$ defined over $\kappa$, which consists in a root $\tau_{0} \simeq \mathbb{P}_{\kappa}^{1}$ with $s$ marked distinct points $\alpha_{1}, \ldots, \alpha_{s}$, and $s$ end components $\tau_{1}, \ldots, \tau_{s}$ attached to the root at $\alpha_{1}, \ldots, \alpha_{s}$ respectively, each of them marked by two points. Choose a deformation $\tilde{\tau}_{0}$ of the marked curve $\tau_{0}$ over $\mathcal{O}: \mathbb{P}_{\mathcal{O}}^{1}$ marked by $\tilde{\alpha}_{1}, \ldots, \tilde{\alpha}_{s}$. At each point $\tilde{\alpha}_{i}$ of $\tilde{\tau}_{0}$ attach a copy $\tilde{\tau}_{i}$ of $\mathbb{P}_{\mathcal{O}}^{1}$ marked by two points $(i=1, \ldots, s)$. The resulting space $\tilde{\tau}$ is a comb over $\mathcal{O}$, which is a deformation of $\tau$.

The restriction of $\varphi$ to each component of $\tau_{i}$ is a cyclic cover branched at two points, $i=1, \ldots, s$. For every given integer $d \geq 1$, there is, up to isomorphism, a unique connected cyclic cover of $\mathbb{P}^{1}$ of degree $d$ branched at two points. Thus each component of $\left.\varphi\right|_{\tau_{i}}$ has a unique deformation to a cover of $\tilde{\tau}_{i}$ branched at two points. The trivial cover given by the restriction of $\varphi$ to $\tau_{0}$ obviously extends to a trivial cover of $\tilde{\tau}_{0}$. The patching datas above 
the point $\alpha_{i}$ between the restrictions of $\varphi$ to $\tau_{0}$ and $\tau_{i}$ uniquely extend to patching datas over $\mathcal{O}(i=1, \ldots, s)$. The result is a HM-admissible cover $\tilde{\varphi}$ of the comb $\tilde{\tau}$.

3.2.3. Second stage. If $\kappa$ is of characteristic $p>0$, use the first stage and denote the generic fiber of $\tilde{\varphi}$ by $\bar{f}$. If $\kappa$ is of characteristic 0 , set $k=\kappa$ and $\bar{f}=\varphi$. In both cases, $\bar{f}$ is a HM-admissible cover of a comb over $k$ and its representing point $[\bar{f}]$ on the moduli space $\overline{\mathcal{H}_{r, G}(\mathbf{C})}$ is a $k$-point in $\overline{\mathcal{H}}$. Similarly as above, fix an embedding $\iota$ of the algebraic closure of the subfield of $\bar{k}$ generated by the branch points of the cover $\bar{f}$ into the field $\mathbb{C}$. The $\mathbb{C}$-cover $\bar{f}^{\iota}$ obtained via this embedding corresponds to a complex point in $\overline{\mathcal{H}}$.

By construction, $\bar{f}^{\iota}$ is a complex HM-admissible cover of a comb $T$ : it is trivial above the root $T_{0} \simeq \mathbb{P}_{\mathbb{C}}^{1}$, has $s$ end components $T_{1}, \ldots, T_{s}$ isomorphic to $\mathbb{P}_{\mathbb{C}}^{1}$, and each of the restrictions of $\bar{f}^{\iota}$ to some connected component above $T_{i}$ is a $\mathbb{C}$-cyclic cover of group $<g_{i}>\subset G$ with inertia canonical invariant $\left(g_{i}, g_{i}^{-1}\right), i=1, \ldots, s$. Furthermore, the elements $g_{1}, \ldots, g_{s}$ generate the (topological) monodromy group of the cover $\bar{f}^{\iota}$, which is the group $G$.

Lemma 3.2 - The $\mathbb{C}$-cover $\bar{f}^{\iota}$ is in the topological closure of some HM-component $\mathcal{H}_{H M}$ of $\mathcal{H}_{r, G}(\mathbf{C})$.

Theorem 1.3 will then follow immediately. Indeed $\overline{\mathcal{H}_{r, G}(\mathbf{C})}$ is locally the quotient of a smooth variety by a finite group [We1] and two distinct components of $\mathcal{H}_{r, G}(\mathbf{C})$ have disjoint boundaries in $\overline{\mathcal{H}_{r, G}(\mathbf{C})}$ (and so components in $\overline{\mathcal{H}_{r, G}(\mathbf{C})}$ are closures of components in $\left.\mathcal{H}_{r, G}(\mathbf{C})\right)$. The representing points of the covers $\varphi$ and $\bar{f}^{\iota}$ are in the same component of $\overline{\mathcal{H}_{r, G}(\mathbf{C})}$; hence they are in the boundary of the same component of $\mathcal{H}_{r, G}(\mathbf{C})$, which from lemma 3.2 is a HM-component.

We give two proofs of Lemma 3.2. The first one uses $\S 2.1$ and the second one $\S 2.2$.

1st proof. The complex comb $T$ can be deformed over the ring $\mathbb{C}\{\{t\}\}$ of Taylor series of positive radius of convergence to a stable curve $\widetilde{P}_{\mathbf{t}}$ of genus 0 with $2 s$ sections $\tilde{x}_{1}, \tilde{y}_{1}, \ldots, \tilde{x}_{s}, \tilde{y}_{s}$ and whose generic fiber is a $\mathbb{P}^{1}$ with $2 s$ marked points $x_{1}, y_{1}, \ldots, x_{s}, y_{s}$. The HM-admissible cover $\bar{f}^{\iota}$ can be extended to a $\mathbb{C}\{\{t\}\}$-cover $\widetilde{f}$ whose generic fiber is a smooth cover of $\mathbb{P}^{1}$ branched at $x_{1}, y_{1}, \ldots, x_{s}, y_{s}$. As all the varieties we consider are of finite type over $\mathbb{C}\{\{t\}\}$, there exists a real number $\rho>0$ such that $\widetilde{f}$ induces an analytic family of covers $\widetilde{f}_{\theta}(0<\theta \leq \rho)$ of $\mathbb{P}^{1}$ defined over $\mathbb{C}$ ramified at $2 s$ points $x_{1}^{\theta}, y_{1}^{\theta}, \ldots, x_{s}^{\theta}, y_{s}^{\theta}$ (the specializations of $\tilde{x}_{1}, \tilde{y}_{1}, \ldots, \tilde{x}_{s}, \tilde{y}_{s}$ at $t=\theta$ ).

We now apply the topological version of Theorem 2.1. The topological fundamental group of the fiber at $\theta$ of $\widetilde{P}_{\mathbf{t}}\left(\right.$ with $\left.\mathbf{t}=\left\{x_{1}, y_{1}, \ldots, x_{s}, y_{s}\right\}\right)$ which we denote below by $\left(\mathbb{P}^{1}\right)^{\theta}$, is constant. There are natural morphisms (defined up to conjugation), for every $\theta \in] 0, \rho[$ : 


$$
\begin{aligned}
& \left.\theta_{i}: \pi_{1}^{\mathrm{top}}\left(T_{i} \backslash\left\{\bar{a}_{i}, \bar{x}_{i}, \bar{y}_{i}\right\}, \xi_{i}\right) \rightarrow \pi_{1}^{\mathrm{top}}\left(\left(\mathbb{P}^{1}\right)^{\theta} \backslash\left\{x_{1}^{\theta}, y_{1}^{\theta}, \ldots, x_{s}^{\theta}, y_{s}^{\theta}\right\}, \xi\right)\right) \quad(i=1, \ldots, s) \\
& \theta_{0}: \pi_{1}^{\mathrm{top}}\left(T_{0} \backslash\left\{\bar{a}_{1}, \ldots, \bar{a}_{s}\right\}, \xi_{0}\right) \rightarrow \pi_{1}^{\mathrm{top}}\left(\left(\mathbb{P}^{1}\right)^{\theta} \backslash\left\{x_{1}^{\theta}, y_{1}^{\theta}, \ldots, x_{s}^{\theta}, y_{s}^{\theta}\right\}, \xi\right)
\end{aligned}
$$

where, as in $\S 2.1, \bar{x}_{i}, \bar{y}_{i}$ are the specialization of $\tilde{x}_{i}$ and $\tilde{y}_{i}$ on the special fiber of $\widetilde{P}_{\mathbf{t}}$ $(i=1, \ldots, s), \bar{a}_{1}, \ldots, \bar{a}_{s}$ are the intersection points of the end components with the root of $T$, and where $\xi_{0}, \xi_{1}, \ldots \xi_{s}$ and $\xi$ are appropriate base points. We also have

- a topological bouquet $\underline{\gamma}^{(i)}=\left\{\gamma_{0}^{(i)}, \gamma_{1}^{(i)}, \gamma_{2}^{(i)}\right\}$ for $T_{i} \backslash\left\{\bar{a}_{i}, \bar{x}_{i}, \bar{y}_{i}\right\}$ based at $\xi_{i}, i=1, \ldots, s$,

- a topological bouquet $\underline{\gamma}^{(0)}=\left\{\gamma_{1}^{(0)}, \ldots, \gamma_{s}^{(0)}\right\}$ for $T_{0} \backslash\left\{\bar{a}_{1}, \ldots, \bar{a}_{s}\right\}$ based at $\xi_{0}$, and

- elements $\sigma_{i} \in \pi_{1}^{\mathrm{top}}\left(\left(\mathbb{P}^{1}\right)^{\theta} \backslash\left\{x_{1}^{\theta}, y_{1}^{\theta}, \ldots, x_{s}^{\theta}, y_{s}^{\theta}\right\}, \xi\right), i=1, \ldots, s$,

such that $\pi_{1}^{\text {top }}\left(\left(\mathbb{P}^{1}\right)^{\theta} \backslash\left\{x_{1}^{\theta}, y_{1}^{\theta}, \ldots, x_{s}^{\theta}, y_{s}^{\theta}\right\}, \xi\right)$ is generated by the elements

$$
\begin{aligned}
& \text { - } \theta_{0}\left(\gamma_{1}^{(0)}\right), \ldots, \theta_{0}\left(\gamma_{s}^{(0)}\right), \text { and } \\
& \text { - } \theta_{i}\left(\gamma_{0}^{(i)}\right), \theta_{i}\left(\gamma_{1}^{(i)}\right), \theta_{i}\left(\gamma_{2}^{(i)}\right), i=1, \ldots, s
\end{aligned}
$$

with the only relations $\theta_{0}\left(\gamma_{i}^{(0)}\right) \cdot \theta_{i}\left(\gamma_{0}^{(i)}\right)^{\sigma_{i}}=1, i=1, \ldots, s$. Furthermore the elements $\theta_{1}\left(\gamma_{1}^{(1)}\right)^{\sigma_{1}}, \theta_{1}\left(\gamma_{2}^{(1)}\right)^{\sigma_{1}}, \ldots, \theta_{s}\left(\gamma_{1}^{(s)}\right)^{\sigma_{s}}, \theta_{s}\left(\gamma_{2}^{(s)}\right)^{\sigma_{s}}$ constitute a topological bouquet of $\left(\mathbb{P}^{1}\right)^{\theta} \backslash$ $\left\{x_{1}^{\theta}, y_{1}^{\theta}, \ldots, x_{s}^{\theta}, y_{s}^{\theta}\right\}$.

As the cover $\bar{f}^{\iota}$ is unramified at each point $\bar{a}_{i}, i=1, \ldots, s\left(\bar{f}^{\iota}\right.$ is HM-admissible), the Branch Cycle Description of the cover $\widetilde{f}_{\theta}$ with respect to this topological bouquet is of the form $g_{1}^{h_{1}},\left(g_{1}^{-1}\right)^{h_{1}}, \ldots, g_{s}^{h_{s}},\left(g_{s}^{-1}\right)^{h_{s}}$. The cover $\widetilde{f}_{\theta}$ is the unique deformation of $\bar{f}^{\iota}$ above the path $\left\{x_{1}^{\theta}, y_{1}^{\theta}, \ldots, x_{s}^{\theta}, y_{s}^{\theta}\right\}(\theta \in] 0,1[)$ and hence is a connected cover of monodromy group $G$. Thus the cover $\widetilde{f}_{\theta}$ is a complex Harbater-Mumford cover corresponding to some point in $\mathcal{H}_{r, G}(\mathbf{C})$, which proves lemma 3.2 .

2nd proof. $§ 2.2$ explains how to construct a family of HM-covers degenerating to a complex HM-admissible cover $f_{1}$. From the addendum to construction, there is no restriction on the degenerate cover $f_{1}$. We take it to be $\bar{f}^{\iota}$. The HM-covers $f_{\theta}(0<\theta<1)$ provided by the construction have then $2 s$ branch points, their group is the group $G$ generated by $g_{1}, \ldots, g_{s}$ and the inertia canonical invariant is the tuple $\mathbf{C}$ consisting of the $s$ pairs of conjugacy classes $C_{i}, C_{i}^{-1}$ of $g_{i}$ and $g_{i}^{-1}, i=1, \ldots, s$. This shows indeed that $\bar{f}^{\iota}$ is in the topological closure of some HM-component $\mathcal{H}_{H M}$ of $\mathcal{H}_{r, G}(\mathbf{C})$. 


\section{Application to Hurwitz towers}

This section is devoted to our application to inverse Galois theory; the previous sections will be used in the special context of G-covers.

Let $\left(G_{n}\right)_{n \geq 0}$ be a projective system of finite groups, given with surjective morphisms $s_{n}: G_{n} \rightarrow G_{n-1}(n>0)$ and, as in $\S 1.2$, let $k$ be a henselian field of characteristic 0 , of residue characteristic either $p=0$ or $p>0$ not dividing any of the orders $\left|G_{n}\right|(n \geq 0)$ and containing all roots of 1 of prime-to- $p$ order. In [DeDes] a construction is given that produces a regular tower $\left(K_{n}\right)_{n}$ realizing the inverse system $\left(G_{n}\right)_{n}$ over $k(T)$ : that is, $\operatorname{Gal}\left(K_{n} / k(T)\right) \simeq G_{n}$, compatibly with the $s_{n}$ and $K_{n} / k$ regular $(n \geq 0)$.

More precisely, [DeDes] explicitly provides a sequence $\left(r_{n}\right)_{n \geq 0}$ of integers $r_{n} \geq 3$ and a sequence $\left(\mathbf{C}_{n}\right)_{n \geq 0}$ of $r_{n}$-tuples $\mathbf{C}_{n}$ of conjugacy classes of $G_{n}$ such that for each $n \geq 0$, the $n$-th level of the tower is (the function field of) a $k$-G-cover $f_{n}$ of group $G_{n}$, with $r_{n}$ branch points and with inertia canonical invariant $\mathbf{C}_{n}$. These sequences can be constructed independently of the field $k$. So [DeDes] leads to the following modular conclusions [DeDes] $\S 4$. There is a tower $\left(\mathcal{H}_{G_{n}, r_{n}}\left(\mathbf{C}_{n}\right)\right)_{n \geq 0}$ of Hurwitz spaces $\mathcal{H}_{G_{n}, r_{n}}\left(\mathbf{C}_{n}\right)(n \geq 0)$, given with algebraic morphisms $\mathcal{H}_{G_{n}, r_{n}}\left(\mathbf{C}_{n}\right) \rightarrow \mathcal{H}_{G_{n-1}, r_{n-1}}\left(\mathbf{C}_{n-1}\right)(n>0)$, which has the following properties. If $k$ is any henselian field as above, there exist projective systems of $k$-points on the tower $\left(\mathcal{H}_{n}\right)_{n \geq 0}$; for example, there exist projective systems of $\mathbb{Q}^{\mathrm{ab}}((x))$-rational points and there exist projective systems of $\mathbb{Q}_{p}^{\text {ur }}$-rational points, for each prime $p$ such that all $G_{n}$ are of prime-to- $p$ order ${ }^{4}(n \geq 0)$. Also there exist projective systems of $\mathbb{R}$-points.

The connection with the current paper is that in [DeDes], the covers $f_{n}$ are, at each level $n$, constructed thanks to the rigid patching techniques $(n \geq 0)$. With remark 1.6, conclude the covers $f_{n}$ can be constructed to be HM-covers; consequently the corresponding points on the Hurwitz space lie in some HM-component. Furthermore, the sequences $\left(r_{n}\right)_{n \geq 0}$ and $\left(\mathbf{C}_{n}\right)_{n \geq 0}$ can be chosen in such a way that $\mathbf{C}_{n}$ is HM-g-complete and so there is a single HM-component, say $\mathcal{H}_{n}$, on $\mathcal{H}_{G_{n}, r_{n}}\left(\mathbf{C}_{n}\right)$, and it is defined over $\mathbb{Q}(n \geq 0)$. The following statement recapitulates the properties of $\mathcal{H}_{n}$.

Theorem 4.1 - Given a projective system $\left(G_{n}\right)_{n \geq 0}$ of finite groups, one can construct a projective system (a tower) $\left(\mathcal{H}_{n}\right)_{n \geq 0}$ of $\mathbb{Q}$-varieties $\mathcal{H}_{n}$, geometrically irreducible, with algebraic morphisms $\psi_{n}: \mathcal{H}_{n} \rightarrow \mathcal{H}_{n-1}$ defined over $\mathbb{Q}$, with the following properties:

(i) For each $n \geq 0$, the $\mathbb{Q}$-variety $\mathcal{H}_{n}$ is an irreducible component of some Hurwitz space $\mathcal{H}_{r_{n}, G_{n}}$ (for some integer $r_{n}>0$ ).

4 The fields $\mathbb{Q}^{\mathrm{ab}}((x))$ and $\mathbb{Q}_{p}^{\text {ur }}$ can even be replaced by the smaller henselian subfields $\mathbb{Q}^{\mathrm{ab}}((x))^{\text {alg }}$ and $\left(\mathbb{Q}_{p}^{\text {ur }}\right)^{\text {alg }}$ of all elements algebraic over $\mathbb{Q}(x)$ and $\mathbb{Q}$ respectively. 
(ii) As a consequence of (i), recall that if $k$ is any field of characteristic 0, existence of $k$-rational points on $\mathcal{H}_{n}$ implies the group $G_{n}$ can be realized as the automorphism group of a $\bar{k}$-G-cover of $\mathbb{P}^{1}$ of field of moduli $k$.

(iii) If $k$ is any henselian field of characteristic 0 , of residue characteristic either $p=0$ or $p>0$ not dividing any of the orders $\left|G_{n}\right|(n \geq 0)$, and containing all roots of 1 of primeto-p order, there exist projective systems of $k$-points on the tower $\left(\mathcal{H}_{n}\right)_{n \geq 0}$. For example, there exist projective systems of $\mathbb{Q}^{\mathrm{ab}}((x))$-rational points and there exist projective systems of $\mathbb{Q}_{p}^{\text {ur }}$-rational points, for each prime $p$ such that all $G_{n}(n \geq 0)$ are of prime-to- $p$ order. (iv) In (iii), the projective systems of rational points have the extra property that at each level $n \geq 0$, the point lies in the no-obstruction locus of $\mathcal{H}_{n}$, that is, where the field of moduli is a field of definition. Consequently, the projective systems of $k$-rational points in question in (iii) correspond to projective systems of $k$-G-covers $X_{n} \rightarrow \mathbb{P}^{1}$, or equivalently, to towers of $k$-regular extensions $K_{n} / k(T)$, realizing the system $\left(G_{n}\right)_{n \geq 0}$.

Remarks 4.2. (a) In general, Hurwitz spaces are coarse moduli spaces and so $k$-rationality of their points $[f]$ only corresponds to $f$ being of field of moduli $k$ but not necessarily defined over $k$. In the previous sections, we do get conclusions about the field of definition being $k$. So some information is lost in stating the results in terms of rational points on moduli spaces as in (iii). Assertion (iv) compensates this loss. We could have instead stated the result in terms of stacks rather than moduli spaces. However in this refined version, the stack-theoretic $\mathrm{H}_{n}$ counterpart of $\mathcal{H}_{n}$ would not be an algebraic variety anymore.

(b) Recall that presence of roots of 1 in the base field $k$ is not just a technical assumption due to the method employed. The result would be false otherwise: for example, the group $\mathbb{Z}_{p}=\lim _{\longleftarrow} \mathbb{Z} / p^{n} \mathbb{Z}$ is not a regular Galois group over $\mathbb{Q}_{\ell}(T)[\mathrm{Se}]$

(c) Another application, to modular towers is developed in [DeDes]. Suppose given a finite group $G$ and a prime number $\ell$ dividing $|G|$ and assume $G$ has a set of generators of order $\rho$ prime to $\ell$. Denote the $\ell$-universal Frattini cover of $G$ by $\ell \tilde{G}$; it is naturally the inverse limit of some projective system $\left({ }_{\ell}^{n} \tilde{G}\right)_{n \geq 0}$. A typical example is this: $G$ is the dihedral group $D_{p}$ of order $2 p, \ell=p$ is an odd prime, $\rho=2$ and the projective system $\left({ }_{\ell}^{n} \tilde{G}\right)_{n \geq 0}$ is the sequence of dihedral groups $\left(D_{p^{n+1}}\right)_{n \geq 0}$, which converges to $D_{p^{\infty}}=\mathbb{Z}_{p} \times{ }^{s} \mathbb{Z} / 2$. Suppose now given a henselian field $k$ of characteristic 0 ; it is not assumed here that $k$ contains roots of 1 . Then the general construction of [DeDes] applies to yield a realization of $\ell \tilde{G}$ by a tower of regular Galois extensions of $k(T)$; furthermore the inertia canonical invariant $\mathbf{C}_{n}$ of the realizing cover at level $n$ consists of a fixed number, say $r$, of conjugacy classes of order $\rho(n \geq 0)$. Again this interprets as the existence of a projective system of $k$-rational points on a certain 
tower of Hurwitz spaces, namely the tower $\left(\mathcal{H}_{r, n_{\ell}^{n}}\left(\mathbf{C}_{n}\right)\right)_{n \geq 0}$. This tower is a modular tower, as constructed by M. Fried $[\mathrm{Fr}][\mathrm{BaFr}]$. As before, the covers used to realize all finite levels ${ }_{\ell}^{n} \tilde{G}(n \geq 0)$ are of Harbater-Mumford type. If in addition, $\mathbf{C}_{0}$ is HM-g-complete, then so are all $\mathbf{C}_{n}$ and so each space $\mathcal{H}_{r,{ }_{\ell}} \tilde{G}\left(\mathbf{C}_{n}\right)$ has a unique HM-component, defined over $\mathbb{Q}$. Conclude as before that the projective system of $k$-rational points mentioned above can be found on a tower of algebraic varieties, geometrically irreducible and defined over $\mathbb{Q}$; furthermore these varieties are here all of the same dimension, namely $r$.

\section{Formal viewpoint}

In $\S 4$, concerning the construction of infinite towers realizing the complete system $\left(G_{n}\right)_{n \geq 0}$, we rely on the paper [DeDes] where a rigid approach is used. The goal of this section is to give an alternate proof using formal geometry and thus to provide a complete formal approach to theorem 4.1 .

Theorem 5.1 - Let $k$ be a henselian field of residue characteristic $p \geq 0$ and containing all roots of 1 of prime-to-p order. Let $\left(G_{n}\right)_{n \geq 0}$ be a projective system of finite groups of prime-to-p order. Then there exists a projective system $\left(f_{n}\right)_{n \geq 0}$ of HM-Galois covers of $\mathbb{P}^{1}$ defined over $k$ and with corresponding Galois groups $\left(G_{n}\right)_{n \geq 0}$.

Proof. We are given a projective system $\left(G_{n}\right)_{n \geq 0}$ with epimorphisms $s_{n}: G_{n} \rightarrow G_{n-1}$. For each $n \geq 0$, choose a generating system $\underline{g}^{(n)}=\left(g_{1}^{(n)}, \ldots, g_{q_{n}}^{(n)}\right)$ of $G_{n}$ such that

$$
\left\{\begin{array}{c}
s_{n+1}\left(g_{j}^{(n+1)}\right)=g_{j}^{(n)}, j=1, \ldots, q_{n} \\
s_{n+1}\left(g_{j}^{(n+1)}\right)=1, \quad \text { for all } j>q_{n}
\end{array}\right.
$$

We denote this pro-generating system by $\underline{g}$.

On the other hand one can construct a projective system $\left(T^{(n)}\right)_{n \geq 0}$ of stable marked curves $T^{(n)}$ over the valuation $\operatorname{ring} \mathcal{O}$ of $k$, whose generic fiber is $\mathbb{P}^{1}$ marked by the set $\mathbf{t}_{n}=\left\{x_{1}, y_{1}, \ldots, x_{q_{n}}, y_{q_{n}}\right\}$ of points of $\mathbb{P}^{1}(k)$ and the special fiber is a comb with roots $T_{0}^{(n)}$ and end components $T_{j}^{(n)}\left(j=1, \ldots, q_{n}\right)$, with morphims of marked curves $t_{n+1}$ : $T^{(n+1)} \rightarrow T^{(n)}(n \geq 0)$ inducing the identity map Id on the generic fiber and inducing the following map on the special fiber:

$$
\left\{\begin{aligned}
\mathrm{Id}: T_{0}^{(n+1)} & \rightarrow T_{0}^{(n)} \\
\mathrm{Id}: T_{j}^{(n+1)} & \rightarrow T_{j}^{(n)}, j=1, \ldots, q_{n} \\
T_{j}^{(n+1)} & \rightarrow \bar{a}_{j}, \text { for all } j>q_{n}
\end{aligned}\right.
$$


where $\bar{a}_{j}$ denotes the intersection point of $T_{j}^{(n+1)}$ with $T_{0}^{(n+1)}$.

From $§ 2.1$ we have morphisms of fundamental groups (defined up to conjugation)

$$
\theta_{j}^{(n)}: \pi_{1}\left(T_{j}^{(n)} \backslash\left\{\bar{a}_{j}, \bar{x}_{j}, \bar{y}_{j}\right\}\right) \rightarrow \pi_{1}\left(T_{\bar{\eta}}^{(n)} \backslash \mathbf{t}_{n}\right)
$$

(and similarly with $j=0$ ) making the following diagrams commutative (up to conjugation)

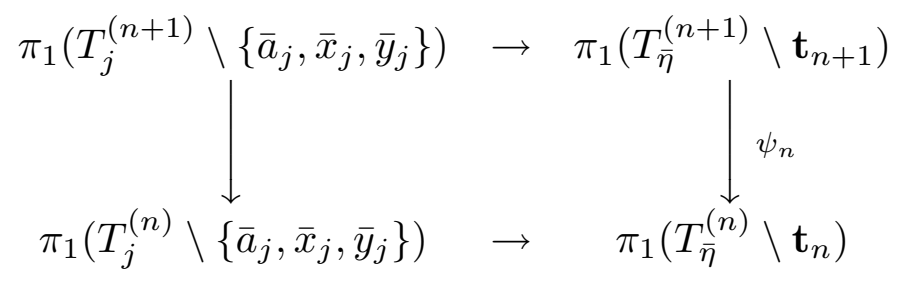

(similarly with $j=0$ ).

For every $n \geq 0$, theorem 2.1 provides a bouquet for $\pi_{1}\left(T_{\bar{\eta}}^{(n)} \backslash \mathbf{t}_{n}\right)$ of the form

$$
\theta_{j}^{(n)}\left(\gamma_{1}^{j}\right)^{\tau_{j}^{n}}, \theta_{j}^{(n)}\left(\gamma_{2}^{j}\right)^{\tau_{j}^{n}}, j=1, \ldots, q_{n}
$$

where $\gamma_{1}^{j}$ (resp. $\gamma_{2}^{j}$ ) is the generator attached to $\bar{x}_{j}$ (resp. to $\left.\bar{y}_{j}\right)$ in a bouquet for $\pi_{1}\left(T_{j}^{(n)} \backslash\right.$ $\left.\left\{\bar{a}_{j}, \bar{x}_{j}, \bar{y}_{j}\right\}\right)$, and $\tau_{j}^{n} \in \pi_{1}\left(T_{\bar{\eta}}^{(n)} \backslash \mathbf{t}_{n}\right)$. Moreover, for each $n \geq 0$, there exists $\omega_{n} \in \pi_{1}\left(T_{\bar{\eta}}^{(n)} \backslash\right.$ $\mathbf{t}_{n}$ ) such that

$$
\left\{\begin{array}{l}
\psi_{n}\left(\theta_{j}^{n+1}\left(\gamma_{1}^{j}\right)^{\tau_{j}^{n+1}}\right)=\left(\theta_{j}^{n}\left(\gamma_{1}^{j}\right)^{\tau_{j}^{n}}\right)^{\omega_{n}}, j=1, \ldots, q_{n} \\
\psi_{n}\left(\theta_{j}^{n+1}\left(\gamma_{2}^{j}\right)^{\tau_{j}^{n+1}}\right)=\left(\theta_{j}^{n}\left(\gamma_{2}^{j}\right)^{\tau_{j}^{n}}\right)^{\omega_{n}}, j=1, \ldots, q_{n} \\
\psi_{n}\left(\theta_{j}^{n+1}\left(\gamma_{2}^{j}\right)^{\tau_{j}^{n+1}}\right)=1, \text { for all } j>q_{n}
\end{array}\right.
$$

From HM-admissible covers of the special fiber of $T^{(n)}$ built from cyclic covers of the end components, one can construct HM- $p^{\prime}$-covers of the generic fiber $T_{\eta}^{(n)}$ of group $G_{n}$ branched at the $2 q_{n}$ marked points, whose branch cycle description in the previous bouquet is

$$
\left(\theta_{j}^{n}\left(\gamma_{1}^{j}\right)^{\tau_{j}}\right) \rightarrow h_{j}^{(n)} \quad\left(\theta_{j}^{n}\left(\gamma_{2}^{j}\right)^{\tau_{j}}\right) \rightarrow\left(h_{j}^{(n)}\right)^{-1}
$$

where $h_{j}^{(n)}$ is a conjugate of $g_{j}^{(n)}, j=1, \ldots, q_{n}$. Moreover one can require that this cover, which is defined over $k^{5}$, has a totally rational fiber (i.e. consisting of $k$-rational points) over some fixed $k$-rational point of the basis; this follows for instance from the fact that the special fiber of the cover is trivial over the root, and so has many totally $\kappa$-rational

5 The part of theorem 2.1 that we use here - existence of extensions over $\mathcal{O}$ of any $\kappa$-admissible cover - does not need the assumption " $\kappa$ algebraically closed". 
fibers, which extend to totally $k$-rational fibers. A consequence of this property is that two such covers which are isomorphic over $\bar{k}$ are already isomorphic over $k$.

Let $\mathcal{S}_{n}$ be the set of $k$-isomorphism classes of such HM-covers of $T_{\eta}^{(n)}(n \geq 0)$. It is a non-empty finite set. Moreover, if $Z \rightarrow T_{\eta}^{(n+1)}$ is a representative of an element of $\mathcal{S}_{n+1}$, the cover $Z / \operatorname{Ker}\left(s_{n}\right) \rightarrow T_{\eta}^{(n+1)}$ is unramified at the $2 q_{n+1}-2 q_{n}$ marked points which specialize on $T_{j}^{(n)}, q_{n}<j \leq q_{n+1}$, and it induces a $G_{n}$-cover $Z / \operatorname{Ker}\left(s_{n}\right) \rightarrow T_{\eta}^{(n)}$ ramified at the $2 q_{n}$ points from $\mathbf{t}_{n}$. The isomorphism class of this cover clearly belongs to $\mathcal{S}_{n}$.

We have constructed a map from $\mathcal{S}_{n+1}$ to $\mathcal{S}_{n}(n \geq 0)$, and the projective limit of the non-empty finite sets $\mathcal{S}_{n}$ is non-empty. An element of this projective limit is a coherent system of HM-covers of groups $\left(G_{n}\right)_{n \geq 0}$.

\section{References}

[BaFr] P. Bayley and M. Fried, "Hurwitz monodromy, spin separation and higher levels of a Modular Tower", Proceedings of the Von Neumann Symposium on Arithmetic Fundamental Groups and Noncommutative Algebra (MSRI 1999), Proceedings of Symposia in Pure Mathematics,70, AMS, ed. by M. Fried and Y. Ihara, (2002), 70-220.

[De] P. Dèbes, Arithmétique et espaces de modules de revêtements, Proceedings of the Number Theory conference in Zakopane, (K. Gyory, H. Iwaniec and J. Urbanowicz ed.), Walter de Gruyter, (1999), $75-102$.

[DeDes] P. Dèbes and B. Deschamps, Corps $\psi$-libres et théorie inverse de Galois infinie, preprint.

[Em1] M. Emsalem, Espaces de Hurwitz, in Arithmétique des revêtements algébriques, Proceedings des Journées "Revêtements" (St-Etienne 2000), Séminaires et Congrès, 5, SMF, (2001), 63-99.

[Em2] M. Emsalem, Groupoïde fondamental de courbes stables, preprint.

[Fr] M. Fried, Introduction to modular towers, in Recent Developments in the Inverse Galois Problem, Contemporary Math., 186, (1995), 111-171.

[Ha] D. Harbater, Galois coverings of the arithmetic line, Lecture Notes in Math. 1240, (1987), $165-195$.

[Li] Q. Liu, Tout groupe fini est groupe de Galois sur $\mathbb{Q}_{p}(T)$, Contemporary Mathematics, 186, (1995), 261-265.

[Mo] L. Moret-Bailly, Constructions de revêtements de courbes pointées, J. Algebra, 240, (2001), 505-534.

[Po1] F. Pop, Half Riemann's existence theorem, Algebra and Number Theory (G. Frey and J. Ritter, eds), de Gruyter Proceedings in Mathematics, (1994), 1-26

[Po2] F. Pop, Embedding problems over large fields, Annals of Math., 144, 1-35, (1996).

[Se] J.-P. Serre, Topics in Galois theory, Notes written by Henri Darmon, Jones and Bartlett Publ., Boston, (1992).

[Vo] H. Völklein, Groups as Galois groups - an introduction, Cambridge Studies in Advanced Mathematics, 53, Cambridge Univ. Press, (1996).

[We1] S. Wewers, Construction of Hurwitz spaces, thesis, (1998).

[We2] S. Wewers, Deformation of tame admissible covers of curves, in Aspects of Galois Theory, London Math. Soc. Lecture Note Series, 256, (H. Völklein, P. Mueller, D. Harbater and J. G. Thompson ed.), Cambridge University Press, (1999), 239-282.

Pierre.Debes@univ-lille1.fr emsalem@agat.univ-lille1.fr

Univ. Lille 1, Mathématiques, 59655 Villeneuve D’Ascq Cedex, France. 